\title{
Efectos de la evaluación de desempeño en la calidad de vida laboral del trabajador: Revisión del tema entre 2008-2018
}

Effects of performance evaluations on the quality of work life of employees: a review from 2008 to 2018

\author{
Sonia Maritza Matabanchoy-Tulcán'1 orcid.org/0000-0002-3681-8440 \\ Karol Melissa Álvarez-Pabón' orcid.org/0000-0002-8234-6341 \\ Oscar David Riobamba-Jiménez1* orcid.org/0000-0001-7194-3338
}

1 Universidad de Nariño. Pasto, Colombia

Matabanchoy-Tulcán SM, Álvarez-Pabón KM, Riobamba-Jiménez OD. Efectos de la evaluación de desempeño en la calidad de vida laboral del trabajador: revisión del tema entre 2008-2018. Univ. Salud. 2019;21(2):176-187. DOI: http://dx.doi.org/10.22267/rus.192102.152

\section{Resumen}

Introducción: La Evaluación de Desempeño (ED) permite el desarrollo personal-profesional y contribuye al mejoramiento de la Calidad de Vida Laboral (CVL) del trabajador. Posibilita la identificación de fortalezas, genera procesos de autoevaluación y mejoramiento en el desarrollo organizacional y su competitividad. Objetivo: Definir el concepto de evaluación de desempeño y evaluar sus efectos en la calidad de vida laboral del trabajador en algunos sectores durante los últimos 10 años. Materiales y métodos: Revisión sistemática de la literatura, como estudio integrativo, observacional y retrospectivo, en el cual se combinaron investigaciones que examinan la misma temática. Se revisaron 55 artículos publicados en las bases de datos Redalyc, SciELO, ScienceDirect y Dialnet. Resultados: Se presentan los criterios de selección, definición, relación entre CVL y ED abordados en los sectores de salud, educación y organizacional. Conclusiones: La ED reconoce el aporte del trabajador al logro de los objetivos organizacionales, teniendo en cuenta la productividad, el desarrollo y la CVL de los colaboradores.

Palabras clave: Evaluación; desempeño; calidad de vida; laboral. (Fuente: DeCS, Bireme).

\begin{abstract}
Introduction: A Performance evaluations (PE) promotes personal-professional development and contributes to the improvement of the Quality of Work Life (QWL) of employees. A PE also enables the identification of strengths, generates self-evaluation processes and improves organizational development and competitiveness. Objective: To define the concept of performance evaluation and evaluate its effects on the quality of work life of employees from some sectors, during the last 10 years. Materials and methods: A systematic literature review using an integrative, observational and retrospective approach that included research studies sharing the same scope. We reviewed 55 articles published in databases such as Redalyc, SciELO, ScienceDirect and Dialnet. Results: We present and discuss the selection criteria, definition, and association between QWL and PE related to health, education and organizational sectors. Conclusions: The PE recognizes the contribution of the employee to the achievement of the organizational objectives and it takes into consideration the productivity, development and maintenance of the QWL of coworkers.
\end{abstract}

Key words: Evaluation, performance, quality of life; working. (Source: DeCS, Bireme).

*Autor de correspondencia 


\section{Introducción}

La administración del talento humano cada día se convierte en un elemento indispensable para alcanzar el logro organizacional(1), en este sentido y considerando al factor humano un componente importante para la organización, es necesario desarrollar mecanismos capaces de reconocer, acompañar y orientar su progreso laboral(2). Así mismo, la gestión de talento humano (GTH), entendida como el proceso centrado en el desarrollo del potencial humano(3), lleva a cabo procesos y prácticas bajo principios fundamentales que norman, regulan y protegen el desarrollo del trabajador en el desempeño de sus funciones, a través de capacitaciones que faciliten el cumplimiento de sus obligaciones en términos de eficacia y eficiencia1. Además, por medio de la selección del personal, la evaluación del desempeño (ED), la formación, el potenciamiento de actitudes, aptitudes, la remuneración justa, la participación activa y directa de trabajadores en la organización, facilita la obtención de la máxima productividad individual y organizacional(1-3).

A través de los procesos de GTH, el trabajador se sentirá, comprometido con la organización, al ser uno de los pilares fundamentales para el logro de los objetivos corporativos; y satisfecho con su labor(1). Esto, a partir de una adecuada selección de personal para el establecimiento del perfil de cargo requerido, la inducción para fortalecer el contrato psicológico del trabajador, los procesos de capacitación, formación y desarrollo para el fortalecimiento de competencias $(2,4)$. Estas prácticas realizadas dentro de la organización aportan en el desempeño laboral de cada integrante 4 ; puesto que, ayuda a trazar objetivos de desempeño, basados en recompensas con el rendimiento; además, establece expectativas claras y apoya el crecimiento del individuo(5), alcanzando un nivel de satisfacción que le permita tener una mejor condición de vida(6).

En este sentido, la GTH se centra en apoyar el desarrollo de las capacidades de los trabajadores, propiciando una gestión por competencias y conocimiento, con el fin de preparar talento humano idóneo capaz de cumplir con el logro de los objetivos organizacionales. La GTH va más allá de controlar y supervisar; sin embargo, también se hace necesario verificar frecuentemente, si las tareas se están ejecutando adecuadamente, para identificar aspectos de mejora a través de la ED(1). Su utilidad y efectividad se focaliza principalmente en la forma de medir el aporte de cada trabajador al logro de los objetivos organizacionales, pensando en la productividad y en el desarrollo profesional del mismo(7); favoreciendo su CVL considerada un indicador de las experiencias humanas en el sitio de trabajo y su grado de satisfacción. Las organizaciones requieren personas motivadas a participar activamente en los trabajos que ejecutan, que sean recompensadas adecuadamente por sus contribuciones, y que su calidad de vida sea un reflejo real del aporte a la sociedad de la cual hacen parte( ${ }^{(8)}$. Por lo tanto, la ED depende principalmente de la existencia de varios métodos y técnicas para realizar este proceso desde los diferentes sectores que permitan el diseño de planes de reconocimiento y mejora en aquellos casos que así lo requieran, pues los trabajadores representan el pilar fundamental de toda empresa.

Con lo anterior, la ED se convierte en un proceso importante en la organización al ser utilizada como una herramienta para la gestión de las perspectivas de desarrollo personal y profesional de las personas. Es por ello, que el objetivo principal del artículo es definir el concepto de evaluación de desempeño y evaluar sus efectos en la calidad de vida laboral del trabajador en los diferentes sectores durante los últimos 10 años. Para dar cuenta de cómo se realizó el trabajo de investigación, este artículo se divide en 3 partes: primero, se describen los materiales y métodos, donde se presentan los criterios de selección de artículos y el proceso; luego, se indican los principales resultados obtenidos de la revisión documental, destacando la conceptualización, la relación entre ED y CVL, los métodos y técnicas empleadas para realizar esta evaluación. Finalmente, la discusión y la conclusión del trabajo realizado.

\section{Materiales y métodos}

El artículo se desarrolló teniendo en cuenta la metodología de revisión sistemática de la literatura, como estudio integrativo, observacional y retrospectivo, en el cual se combinaron investigaciones que examinan la misma temática. Se revisó 55 artículos publicados en las bases de datos Redalyc, Scielo, ScienceDirecty Dialnet. Como criterios de selección se tuvo en cuenta el año de publicación, desde el 2008 hasta el 2018; el idioma, español o inglés y por sector de investigación: salud, educativo y organizacional. Se consideraron únicamente 
investigaciones finalizadas, con referencias disponibles y acceso al texto completo. Las palabras clave utilizadas fueron: "evaluación", "desempeño", "calidad de vida laboral", en la búsqueda en español y términos similares como: "evaluation", "performance", "quality of work life", en inglés.

En el desarrollo de la revisión sistemática se realizó: 1), objetivo de investigación; 2), especificación de los criterios de inclusión de los estudios; 3 ), formulación de un plan de búsqueda de la literatura; 4), búsqueda de los artículos en las diferentes bases de datos; 5), clasificación de cada estudio de acuerdo a los criterios establecidos; 6), evaluación de la calidad de los estudios y sistematización de la información; 7) interpretación y presentación de los resultados. En este sentido, se extrajo de estos artículos una definición global, los efectos de la ED en la calidad de vida de los trabajadores de manera general y por cada sector, los métodos y técnicas utilizadas para evaluar el desempeño de los trabajadores de acuerdo al sector de investigación.

\section{Resultados}

\begin{abstract}
Año y país de publicación
De los 55 artículos seleccionados que cumplieron con los criterios de selección establecidos, se evidencia mayor número de publicaciones en los años 2013 y 2015 con un total de 8 investigaciones en cada año, donde el país que predomina es Estados Unidos en el 2013 y España y Colombia en el 2015. El año que registra menor número de publicaciones es 2009 con un total de 2 investigaciones realizadas. A nivel general, Colombia es el país que presenta el mayor número de publicaciones, seguido de México. La publicación de artículos relacionada con el tema ha sido continua y se registran publicaciones en diferentes partes del mundo. (Tabla 1.)
\end{abstract}

Tabla 1. Publicaciones relacionadas con ED y CVL según año y país

\begin{tabular}{|c|c|c|c|c|c|c|c|c|c|c|c|c|c|c|c|c|c|}
\hline Año/País & 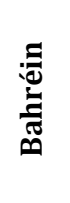 & $\begin{array}{l}\overline{\bar{g}} \\
\bar{D} \\
\bar{D}\end{array}$ & 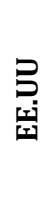 & 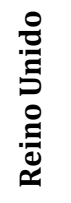 & 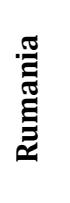 & $\begin{array}{l}\frac{\pi}{8} \\
\frac{8}{2}\end{array}$ & $\underset{\Xi}{\stackrel{\Xi}{\Xi}}$ & 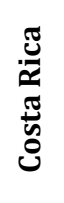 & 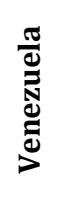 & $\stackrel{0}{\overparen{Z}}$ & 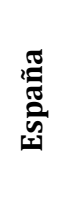 & 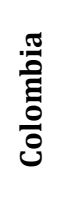 & 气̊ & $\begin{array}{l}\stackrel{\dot{x}}{x} \\
\substack{\mathbb{0} \\
\Sigma}\end{array}$ & 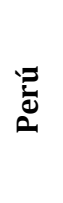 & 氖 & 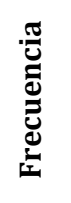 \\
\hline 2008 & & & 1 & & & & & & 1 & & & 1 & & 2 & & & 5 \\
\hline 2009 & & & & & & & & & & & & 1 & & & 1 & & 2 \\
\hline 2010 & & & & & & & & & 1 & 1 & 1 & 1 & 1 & & & & 5 \\
\hline 2011 & & 1 & & & & & & & & 1 & 2 & & & & & & 4 \\
\hline 2012 & 1 & & & 1 & & & & & & & & 1 & 1 & 2 & & & 6 \\
\hline 2013 & & 1 & & & & & & 1 & 1 & & 2 & 2 & & 1 & & & 8 \\
\hline 2014 & & & & & & & & 1 & 1 & & & 1 & & & & & 3 \\
\hline 2015 & & & 2 & & 1 & & & & & & 1 & 1 & 1 & 1 & 1 & & 8 \\
\hline 2016 & & & 1 & & & 2 & & & & & 1 & & 2 & 1 & & & 7 \\
\hline 2017 & & & & & & & 1 & & & 1 & & & & 2 & & & 4 \\
\hline 2018 & & & 1 & & & & & & & & & 2 & & & & 1 & 4 \\
\hline Total & 1 & 2 & 5 & 1 & 1 & 2 & 1 & 2 & 4 & 3 & 7 & 10 & 5 & 9 & 2 & 1 & 56 \\
\hline
\end{tabular}

\section{Idioma del artículo y sectores de investigación}

La mayoría de los artículos están publicados en idioma español, seguidos de inglés. Se identifica 3 sectores de investigación, donde se destaca un número mayor en el sector organizacional con un total de 31 artículos, seguido por el sector educativo con un total de 14 y el sector salud con 11, como se presenta en la Tabla 2.
Tabla 2. Sector de investigación según el idioma

\begin{tabular}{ccccc}
\hline \multirow{2}{*}{ Idioma } & \multicolumn{3}{c}{ Sector de investigación } & \multirow{2}{*}{ Total } \\
\cline { 2 - 4 } & Organizacional & Educativo & Salud & \\
\hline Español & 22 & 14 & 9 & $\mathbf{4 5}$ \\
Inglés & 9 & - & 2 & $\mathbf{1 1}$ \\
Total & $\mathbf{3 1}$ & $\mathbf{1 4}$ & $\mathbf{1 1}$ & $\mathbf{5 6}$ \\
\hline
\end{tabular}




\section{Definición de evaluación de desempeño}

Existen múltiples definiciones de ED según el año y el sector de investigación; sin embargo, desde 1978 se consideró como una base para la toma de decisiones administrativas relacionadas con la promoción de los trabajadores, transferencias y recompensas, así como para la identificación de necesidades, retroalimentación del desempeño y desarrollo personal $^{9}$. En este sentido y teniendo en cuenta las revisiones realizadas, la ED es un proceso de recolección de información, que permite medir y evaluar en el trabajador(10,11), por un lado, el grado de cumplimiento de las funciones y responsabilidades establecidos por la institución (12) y por otro, la calidad con que se lleva a cabo la función en términos de rendimiento $(13,14)$ y los logros obtenidos en un tiempo determinado(15,16), realizando una valoración que identifique las fortalezas y oportunidades de mejora en la función(17). El objetivo de la ED es hacer una estimación cuantitativa y cualitativa(18) del grado de eficacia con que las personas llevan a cabo las actividades, objetivos y responsabilidades de sus puestos de trabajo ${ }^{(19,20)}$. Además, permite apreciar la integración de las personas en sus puestos, mejorar su actuación futura y, con ello, elevar el nivel de desempeño mediante la retroalimentación, el reconocimiento de fortalezas, debilidades y la capacitación coherente con la estrategia organizacional(21,22).

$\mathrm{Su}$ desarrollo en las organizaciones posibilita el mejoramiento del rendimiento laboral, el aumento de las expectativas de comunicación, el fortalecimiento del potencial de los trabajadores y la optimización de la calidad del servicio prestado(23).

\section{Evaluación de desempeño y calidad de vida del trabajador}

Desde 1920 se ha buscado reestructurar positivamente la concepción del trabajador, como parte activa e importante de la empresa y se ha sugerido incorporar dimensiones éticas en los sistemas de evaluación de desempeño como forma integrativa de la dimensión personal y laboral, con el objetivo de que el comportamiento ético en el trabajo sea relevante y gratificante(24). Por ello, se han generado estrategias que evalúan humana $y$ sistemáticamente el desempeño de los colaboradores, de tal manera que puedan apropiar los conocimientos y mejorar su propio entorno a partir de las experiencias en su lugar de trabajo(25).
En este sentido, es menester hablar de la calidad de vida laboral, como una meta que permite establecer conjuntamente compromisos organizacionales que garanticen mejorar las condiciones de trabajo; como un proceso que se encamina a conseguir satisfactoriamente los objetivos trazados a través de la participación activa de sus colaboradores, propiciando altos niveles de satisfacción laboral, e infundiendo la idea básica de la CVL, reconociendo la dignidad humana de los miembros de la organización(26).

Así mismo, es considerada como el grado de percepción y apropiación que tiene el trabajador de su experiencia de manera objetiva y subjetiva, relacionado con adecuadas condiciones laborales como la seguridad en el trabajo, aspectos contractuales y de recompensa, desarrollo personal y profesional, resultados organizacionales, entre otras $(27,28)$. Como resultado, la CVL fomenta el cuidado de la salud, las actitudes positivas entre los miembros de la organización en todos sus niveles, óptimas condiciones personales y de trabajo, además de identificar las dificultades que requieran la toma de decisiones, pues de ello depende la calidad de desempeño y los resultados organizacionales(29).

Teniendo en cuenta lo anterior y con la revisión documental realizada, es posible evidenciar la importancia de la ED en el fortalecimiento de la CVL, pues detecta necesidades de capacitación, identifica a personas clave en la organización, las potencialidades de los evaluados, favorece el desarrollo personal a partir de la retroalimentación y autoevaluación(13); además, facilita la creación de ambientes laborales adecuados para cada uno de los trabajadores en todos sus niveles(24).

\section{Efectos de la evaluación de desempeño en la calidad de vida laboral del trabajador por cada sector de investigación}

\section{Sector salud}

Ha generado grandes avances en los procesos de reforma e inclusión del tema de recursos humanos y su adecuada gestión en cada institución, pues el factor humano es indispensable para el cumplimiento de los objetivos organizacionales, la buena atención en salud y el desarrollo profesional(1,30).

Un ejemplo de ello es la enfermería, pues al ser una profesión que se caracteriza por el cuidado del ser 
humano y su comunidad, es importante el desarrollo de actitudes y capacidades en pro del ser y del hacer, formando un profesional capaz, humano, solidario, crítico, responsable y creativo en la ejecución de su quehacer social-profesional en aras de una trasformación de la realidad y de sí mismos. Por esta razón, actualmente es prioridad la calidad de la atención en el sector salud, pues el desempeño de los profesionales a cargo garantiza la efectividad del cuidado y la mejora de la salud de los usuarios(25). Según el estudio realizado por Gabriel, et al.(30), se reconoce la necesidad de evaluar cuantitativa y cualitativamente este sector para identificar detalladamente la calidad de la atención prestada al paciente, aún más al encontrar escasez de bibliografía sobre evaluación, específicamente, sobre resultados de la asistencia, o indicadores de resultados para la evaluación del servicio en el personal de enfermería(30). Por esto, se han enfocado en la identificación de necesidades de aprendizaje, la elaboración de estrategias de capacitación, el establecimiento de un sistema de seguimiento a los procesos realizados e incentivar los mecanismos de auto-superación, con el fin de perfeccionar las capacidades técnico-profesionales y la transformación de los servicios de salud(30,31). De igual forma, para que este sector logre un buen desarrollo empresarial, es indispensable formar a talento humano idóneo y capaz de influir positivamente en el servicio a partir de la ejecución adecuada de una ED; pues, se considera actualmente como un elemento indispensable para la Gestión del Talento Humano ${ }^{(1,32) .}$

Del mismo modo, al relacionar la ED con la CVL en el sector salud, se identifican percepciones positivas de los colaboradores frente al significado del trabajo y el compromiso laboral, esto debido a que ellos mismos (tras el desarrollo de programas basados en la ED), no solo perciben la posibilidad de desarrollo y progreso al vincularse psicológicamente con la organización (compromiso), sino también que sus resultados aportan al cumplimento de los objetivos organizacionales; generando a su vez motivación e identificación organizacional, variables que influyen en su bienestar y CVL ${ }^{(32)}$. Por lo tanto, el desarrollo de este proceso en el sector salud deberá posibilitará la optimización de competencias laborales, estimulará la superación y el desarrollo cualitativo, mejorando la calidad de la salud y la satisfacción de sus usuarios(33).

\section{Sector educativo}

La ED tiene como objetivo mejorar la calidad del proceso de enseñanza-aprendizaje, a partir del reconocimiento de fortalezas y debilidades de los evaluados y del desarrollo de planes pedagógicos y profesionales de mejora(34,35). En este sentido, la ED resulta ser una herramienta de retroalimentación que permite fortalecer procesos didácticos, metodológicos y pedagógicos; es decir, hacer de esto un factor esencial para la mejora de la educación y de la calidad de vida de la comunidad educativa( $(36,37)$. Sin embargo, este proceso de evaluación ha sido entendido, en algunas ocasiones, como un instrumento de control, de inhibición de la libertad profesional y generador de conflictos éticos, políticos, económicos y sociales; influyendo negativamente en el estado de ánimo y el desempeño académico-laboral de los docentes (36,38). Entre los motivos de malestar se resalta la entrega de incentivos a quien no lo merece de acuerdo a la percepción de los mismos trabajadores, teniendo como referencia su desempeño(38); por lo tanto, si estas dificultes se ven relacionadas, directa o indirectamente, con factores como el aumento de la exigencia académica-laboral y la disminución de sueldos, puede afectar negativamente en la productividad y satisfacción del docente, desencadenando en estrés laboral; el cual se entiende como el conjunto de respuestas fisiológicas, comportamentales, emocionales y cognoscitivas, derivadas del proceso de adaptación a las condiciones de trabajo y de la percepción de insuficiencia de los recursos de afrontamiento para esta situación $(39,40)$.

Algunos docentes reconocen que la ED constituye una fuente de estrés, debido a que, por lo general, es realizada por los alumnos y genera en ellos cierto grado de escepticismo ante lo crítico y subjetivo de los resultados. Esta presión por evitar malas apreciaciones y consecuentemente malos resultados en su evaluación, aumenta la posibilidad del desarrollo de enfermedades y manifestaciones psicosomáticas desencadenadas por el estrés ${ }^{(41,42) \text {. }}$

Por lo anterior, es necesario la construcción de una estrategia de ED que no genere temor ni rechazo, no vigile, controle, castigue, excluya, sino más bien perfeccione la profesión docente a través de la integración dialéctica del saber, saber hacer, saber ser y del saber estar(36,43). En este proceso de construcción, es importante mencionar la vinculación del sector salud y el educativo en la ED, pues para ellos resulta fundamental la mejora de la calidad 
educativa en salud para garantizar la calidad de vida de los pacientes, una formación educativa de calidad y la optimización del nivel de los hospitales y universidades(41,42).

\section{Sector organizacional}

La ED es un proceso que evalúa el progreso hacia el logro de los objetivos preestablecidos $(44,45)$; valora de forma sistemática y objetiva el desempeño de los trabajadores, identificando fortalezas y debilidades en los mismos(12,21), con el fin de elaborar y ejecutar un plan individual de capacitación y desarrollo(22) que genere oportunidades de mejora en la CVL; esto, destaca en el trabajador, un ser humano con grandes potencialidades y posibilidades para avanzar en su crecimiento personal, laboral y social(46). Uno de los medios para lograr este fin es el trabajo, a través de él se obtiene el sustento económico que satisface las necesidades de autorrealización a cambio de un espacio y tiempo relevante en la vida de las personas; éste se puede percibir de dos maneras según el trabajador: como una actividad positiva que le permite construir identidad social dando sentido a la vida en comunidad y elevando el crecimiento personal y profesional; o, como algo indeseado, desarticulado y desprovisto de significados, burocratizado, rutinario, fatigoso, opresivo o incompatible con la vida social y familiar. Estas dos concepciones traen consecuencias positivas o negativas, si éste se siente en un ambiente agradable aumentará la probabilidad de obtener calidad de vida personal, social y familiar, aunque sean esferas distintas y en ellas se desempeñen papeles diferentes; si por el contrario, su percepción es negativa, puede aumentar el riesgo de padecer enfermedades laborales como el estrés(47). Existen varios métodos para evaluar el desempeño de los trabajadores según el sector de investigación (Tabla 3).

Tabla 3. Métodos para evaluar el desempeño según el sector

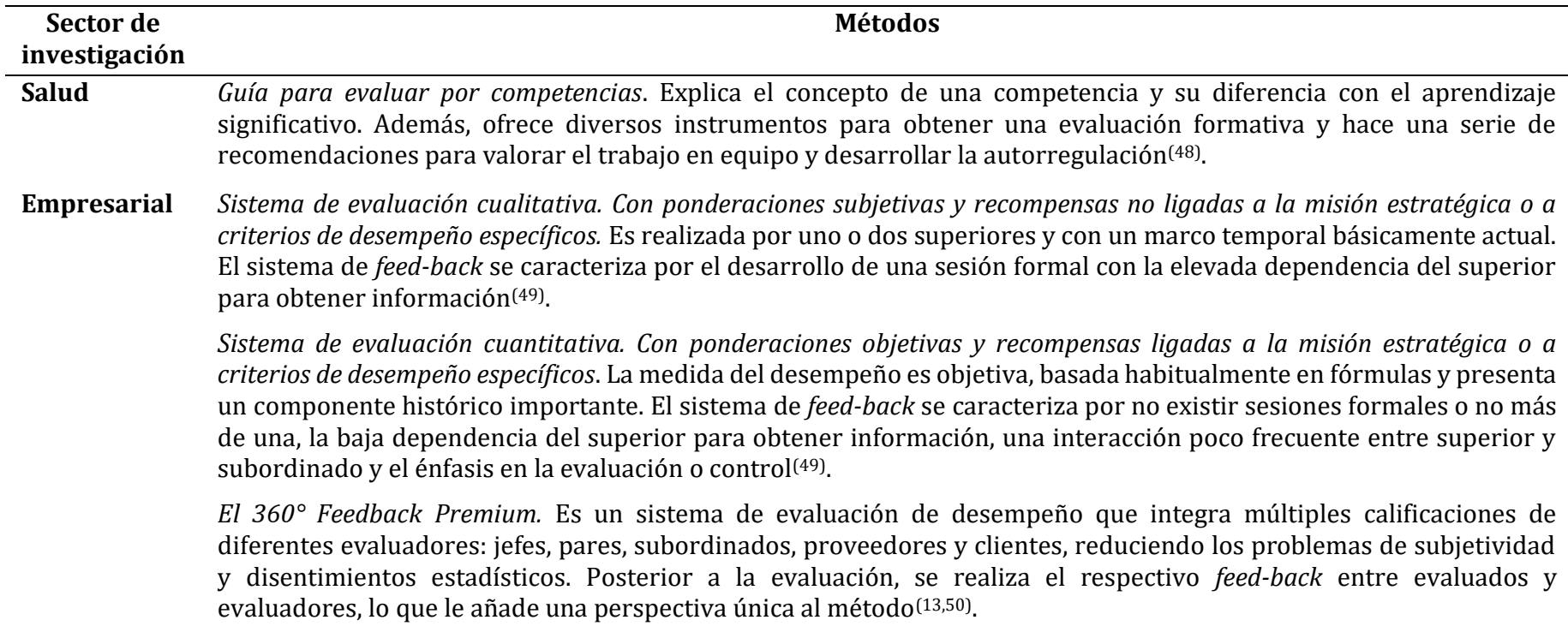

Educativo

Modelo centrado en el perfil del maestro. Se evalúa al docente de acuerdo con el grado de concordancia con los rasgos y características de un perfil previamente establecido(51).

Modelo centrado en los resultados obtenidos. Evalúa al maestro a través de los resultados obtenidos por sus estudiantes(51).

Modelo centrado en el comportamiento del docente en el aula. En la evaluación se identifican aquellos comportamientos del profesor relacionados con los logros de los alumnos y la capacidad del docente para crear un ambiente favorable para el aprendizaje en el aula(51).

Modelo de evaluación a través de portafolio. Implica una recopilación selectiva de materiales, informes y trabajos elaborados por el profesor de manera individual o colectiva durante un periodo de tiempo destinado a observar el desempeño personal. Éstos se enfocan en la planeación o evaluación del proceso de enseñanza-aprendizaje de sus alumnos(51).

Modelo de práctica reflexiva. Consiste en desarrollar, conjuntamente con los maestros, una reflexión supervisada acerca de su práctica. Su objetivo es coadyuvar a elevar la calidad de la educación, reconociendo e impulsando la profesionalización del magisterio, y estimulando a los docentes que cumplen con los requisitos establecidos y obtienen mejores logros en su desempeño a través del mejoramiento de sus condiciones de vida $(36,51)$. 
Cada sector cuenta con métodos que permiten realizar la ED de los trabajadores de manera global y detallada, obteniendo resultados cuantitativos y cualitativos según sea la preferencia de la organización y responda a sus objetivos. Desde el sector organizacional se evidencian métodos correspondientes a la evaluación realizada por el jefe en colaboración con otro personal capacitado para dicha evaluación, con el objetivo de obtener información exhaustiva del desempeño del trabajador en sus funciones diarias; estos métodos, permiten contar con una calificación objetiva a partir de resultados cuantitativos que no implican la intervención directa de los jefes o resultados subjetivos. La variedad de métodos en cuanto a resultados, amplía la posibilidad de obtener información significativa que se ajuste a lo esperado por la organización, garantizando el cumplimiento de sus objetivos y el desarrollo eficaz y eficiente del trabajador.

Desde el sector educativo, se evidencian diferentes métodos que permiten evaluar una parte específica del desempeño de los docentes, permitiéndoles reflexionar sobre su quehacer, contar con la evaluación de compañeros y alumnos, la evaluación de su perfil de acuerdo a las competencias buscadas por la institución, su desempeño en el aula, los avances académicos de sus estudiantes, entre otros. Esto permite realizar específicamente una evaluación detallada del desempeño del docente, que asegura tanto el cumplimiento de los logros esperados por la institución como el crecimiento personal y profesional del mismo.

Desde el sector salud, se evidencia el empleo de una guía que brinda información sobre las competencias que un profesional de la salud debe tener al momento de desempeñarse laboralmente. Dicha guía es de beneficio para la organización que hace uso de ella, pues garantiza identificar cuán capacitado está el personal para la ejecución de sus funciones, teniendo en cuenta las competencias profesionales con las cuales deben contar.

\section{Técnicas para la evaluación del desempeño}

Se mencionan varias técnicas utilizadas para evaluar el desempeño de los trabajadores. A nivel general, entre las técnicas más utilizadas se relacionan en la Tabla 4.

Tabla 4. Técnicas y/o instrumentos para evaluar el desempeño a nivel general

\begin{tabular}{ll}
\hline Técnica y/o instrumento & \multicolumn{1}{c}{ Definición } \\
\hline Observación directa & $\begin{array}{l}\text { Esta técnica de investigación consiste en observar el fenómeno, a evaluar atenta y personalmente y } \\
\text { registrar la información para su posterior análisis. Esta técnica tiene como ventaja el registro de hechos } \\
\text { reales y agrupar situaciones no reproducibles }(51) .\end{array}$
\end{tabular}

Entrevista

Técnica que permite recoger datos; se define como una conversación con un propósito determinado, distinto al hecho de conversar. Se trata de un instrumento técnico que adopta la forma de un diálogo coloquial, a fin de obtener respuestas verbales a las interrogantes planteadas sobre la situación propuesta(22,51).

\section{Autoevaluación}

Auditorías

Escalas gráficas

Selección forzada

Investigación de campo
A través de esta técnica se brinda a los colaboradores herramientas teórico-prácticas que les permita el establecimiento de planes de mejora en su organización o puestos de trabajo. Además, los orienta a la comprensión de conceptos, principios y métodos de gestión en pro de la innovación organizacional(51).

Hace referencia a un examen crítico, sistemático y representativo de la información disponible en una organización, la cual es realizada por un experto a través del uso de técnicas determinadas y que tiene como propósito emitir una opinión profesional sobre los procesos organizaciones, tomar decisiones y mejorar el sistema(4).

Permite evaluar el desempeño de los colaboradores a través de factores definidos previamente. Estos factores definen en cada colaborador capacidades, actitudes, aptitudes y cualidades en la organización. Existen tres tipos de escalas gráficas: continuas, semi-continuas y discontinuas(22,52).

Evalúa el desempeño de los colaboradores mediante frases descriptivas relacionadas a tipos de desempeño individual. El evaluador deberá elegir una o dos frases que más se aproximen a la explicación del desempeño del trabajador evaluado(52).

La evaluación es realizada a la alta gerencia con asesoría de un especialista en este campo. Se indaga y se evalúa el desempeño de sus trabajadores a cargo a través de una entrevista de evaluación y su respectivo seguimiento a los acuerdos de mejora planteados conjuntamente(52). 
Incidentes críticos

Listas de verificación

Escalas de Valoración de Conductas con Anclajes Conductuales (BARS)
Esta técnica busca identificar y comprender conductas extremas o críticas a través de la recolección de observaciones directas del comportamiento del colaborador y que permita determinar las prácticas laborales más y menos efectivos; y así dar solución a los problemas prácticos de la organización(52).

Consiste en la elaboración o utilización de una lista de oraciones que describen el desempeño del colaborador dentro de la organización y que son evaluados por una persona calificada de la misma empresa o externa a ella. Esta persona asigna un valor a las distintas oraciones, de acuerdo a la importancia que presupone cada una(52).

Técnica de evaluación de conductas relacionadas con el trabajo caracterizado por usar graduaciones de niveles de eficiencia y una tipificación conductual. Posee una alta fiabilidad, convirtiéndolo en uno de los tipos más utilizados en los ámbitos laborales Entre sus ventajas se encuentran la mejora del sistema evaluativo, la reducción del efecto halo, un feedback más efectivo de los valoradores a los evaluados y una mayor validez de contenido, que permita la mejora organizacional y la motivación de los colaboradores(15,53).
Teniendo en cuenta lo anterior, se reconoce la falta de un consenso en la forma de evaluar el desempeño y en los efectos que existirán en el sistema de evaluación, producto de la internalización que la organización debe hacer, de manera obligatoria, a circunstancias y hechos concretos que producen cambios que se están desarrollando en el entorno(3). Es por ello, que se recomienda no centrarse en una sola estrategia de ED, sino más bien, conocer la organización y utilizar las técnicas que más convengan con el fin de generar resultados objetivas y completos que permitan fortalecer los procesos organizacionales y personales. Se destacan los grandes aportes en una evaluación objetiva y completa que podría generar la aplicación de la técnica BARS, pues, además de permitir una evaluación y retroalimentación ágil y completa, su aplicación contribuye al control de varios factores adversos que puedan influir negativamente en la satisfacción de necesidades organizacionales y elaborar planes de mejora. Como muestra de ello, algunas investigaciones destacan los beneficios que la escala le otorga a los investigadores en comparación a otras escalas, pues muestra específicamente qué conductas deberían o no presentarse en el puesto de trabajo y cómo éste podría fortalecer algunos aspectos que se identifiquen; además de que se evalúa de manera conjunta aspectos como la motivación, el conocimiento técnico y las habilidades necesarias que le permitirán desplegar todo su potencial en su puesto de trabajo y hacer una evaluación más completa y eficaz ${ }^{(37,50)}$.

\section{Discusión}

El factor humano es un componente importante para el logro de los objetivos organizacionales, por este motivo se han creado mecanismos que permiten reconocer, acompañar y orientar el progreso laboral(1,2), a través de un proceso conocido como Gestión del Talento Humano (GTH), el cual se centra en el desarrollo del potencial humano ${ }^{3}$. Entre los procesos que se llevan a cabo y que facilitan la obtención de la máxima productividad individual y organizacional, se encuentra la selección del personal, la formación, el potenciamiento de actitudes, aptitudes, la remuneración justa, la participación activa y directa de trabajadores en la organización y la evaluación de desempeño (ED) ${ }^{(1-3)}$, siendo este último el principal énfasis del artículo.

En este sentido y teniendo en cuenta los estudios encontrados, se evidencia a través del tiempo en los diferentes sectores sobre ED donde se evidencia características similares entre todas las definiciones y se concluye que la ED es un proceso de recolección de información que permite evaluar cuantitativa y cualitativamente el grado de cumplimiento y calidad con que las personas llevan a cabo las actividades, objetivos y responsabilidades establecidos por una organización. En este se conocen las fortalezas y los aspectos de mejora para trabajar sobre ellos e incrementar el desempeño del trabajador y los resultados de la empresa. Si bien se ha estudiado la ED a lo largo del tiempo, en el año 1978 cuando se empezó a considerarla como una base para la toma de decisiones administrativas relacionadas con la promoción de los trabajadores, transferencias, recompensas, e identificación de necesidades, retroalimentación del desempeño y desarrollo personal(9), se evidencia que dicha definición aún prevalece entre todas las demás.

La ED es un proceso aplicado en los sectores: organizacional, educativo y salud, y cada uno de ellos utiliza diferentes métodos y técnicas que permiten evaluar el desempeño de los trabajadores; en este 
sentido, en el sector organizacional se mencionan métodos cuantitativos y cualitativos según sea la necesidad de la organización, con el fin de medir el aporte de cada trabajador en relación a los objetivos, estándares y expectativas organizacionales(4), mediante valoraciones y retroalimentaciones como fuente de motivación(13) para la obtención de resultados de acuerdo a la responsabilidad del trabajador(2).

En el sector salud, se menciona la guía para evaluar competencias utilizando el estudio de caso con el fin de hacer del profesional un ser competente; cumpliendo con lo planteado por Gabriel, et al.(30), al referir que la ED profesional se realiza en el lugar de trabajo y en una situación de la vida real, con el fin de verificar el comportamiento del profesional en el momento de realizar su quehacer laboral diario; a partir de lo observado se obtiene información relevante del desempeño del trabajador para identificar aspectos de mejora y fortalezas que le permitan al empleador tomar decisiones para el buen cumplimiento de los objetivos organizacionales.

En el marco de los procesos de mejora continua del sector salud, la ED representa no solo el reconocer aquellas dificultades y aspectos por mejorar que tiene el personal en cada institución, sino también el establecimiento de procesos organizacionales constructivos y de desarrollo a nivel personal, profesional y social. Frente a esta postura, Matabajoy, et al.(32), resaltan la importancia que el personal de salud otorga a la detección de necesidades para el desarrollo de programas de capacitación y evaluación que permitan la apropiación y aplicación de conocimientos a su trabajo. De esta forma, significa una transformación indispensable que llevaría a la humanización del servicio de salud, haciendo de éste sea de alta calidad y que ofrezca garantías de un adecuado bienestar general para los usuarios y de calidad de vida para los colaboradores. Cabe resaltar que si bien se identifica la relación entre ED y CVL, ésta se enfoca en el cumplimiento de funciones y no en el desarrollo personal del trabajador(32). Más aún, esto favorece en cierta medida en la formación del trabajador como miembro activo e integral de la empresa a partir de la retroalimentación, identificando sus potencialidades y debilidades que influirán en su desempeño a nivel general y en la creación de entornos laborales saludables(13).
En el sector educativo, se mencionan las autoevaluaciones, evaluaciones por pares $\mathrm{y}$ estudiantes; además de evaluar el perfil del maestro, sus resultados obtenidos, su comportamiento en el aula y la reflexión de su labor(18). Ante esto, es claro que la evaluación docente fomenta y favorece el perfeccionamiento del profesorado en su labor social y educativa, a partir de la reflexión y de la mejora continua de la calidad, al tratarse de un proceso que permite valorar de manera sistemática y continua el conjunto de actitudes, rendimiento y comportamiento laboral del docente en su compromiso de formar y educar integralmente a sus estudiantes $(37,54)$; evaluando el grado de cumplimiento y la calidad con que se llevan a cabo las funciones y responsabilidades establecidas por la institución. Así mismo, se tiene en cuenta los programas de compensación económica, de acreditación institucional y el desarrollo de políticas públicas de evaluación, en los que predomina la opinión de los estudiantes recolectada a través de

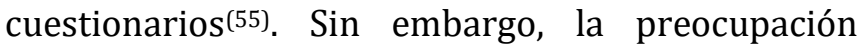
progresiva por las consecuencias a la salud que la ED podría generar a los docentes, evidencia que la sociedad ha aprehendido la idea contraproducente de que evaluar es controlar el desarrollo individual y rendimiento grupal(56). Por ello, es necesario el desarrollo de estrategias de evaluación que permitan obtener resultados significativos y resignificar el valor de la ED en el área escolar.

A nivel general, se menciona algunas técnicas y métodos empleados en los sectores de investigación sin distinción alguna, que evalúan el desempeño del trabajador en la organización; sin embargo, la no homogenización en la evaluación se debe a las particularidades y mecanismos propios de cada empresa, pues cada una precisa satisfacer necesidades en momentos específicos, lo que conlleva a utilizar una u otra técnica dependiendo del tiempo y resultados esperados $(4,57)$. En este sentido, se reconoce aspectos tanto positivos como negativos de cada técnica y método en el proceso de la ED; de igual manera, se resaltan dos técnicas (BARS y $360^{\circ}$ ), que por su aplicación, abordaje y resultados son recomendables para el desarrollo de capacidades y habilidades interprofesionales en aras del progreso organizacional(43,58).

De esta forma, al realizar una ED es posible que el trabajador alcance un desarrollo personal y profesional de acuerdo con sus aportes en la 
consecución de los objetivos organizacionales, pensando no sólo en la productividad de la empresa sino en el progreso personal-laboral del trabajador. $\mathrm{Su}$ utilidad se refleja en el mejoramiento de debilidades evidenciadas en su evaluación y en la posibilidad de la renovación organizacional aplicada a los empleadores y principalmente a colaboradores quienes constituyen la base de toda empresa(59,60). Además, la ED es útil a la hora de identificar falencias en aspectos importantes en la empresa, el desarrollo adecuado de funciones, de un clima organizacional y el conocer el desempeño integral del colaborador(7). De igual forma, permite que la organización mantenga políticas de mejora continua, aprendizaje, crecimiento $\mathrm{y}$ fortalecimiento organizacional que vayan dirigidos a enfrentar nuevos retos empresariales, cumplimiento de metas y resultados, la transformación del personal como socios estratégicos y la adaptación a un cambio continuo(7).

\section{Conclusiones}

De esta forma, la ED se aplica en todas las empresas sin distinción de sector (organizacional, educativo, salud), cada uno utilizando diferentes métodos y técnicas que permiten evaluar el desempeño de los trabajadores e identificar su grado de eficacia dentro del cumplimiento de sus funciones. Esto es posible a través de las investigaciones encontradas en diversos campos, países, versiones, idiomas y periodo de tiempo. Sin embargo, al ser un tema de interés, es indispensable reconocer la necesidad de continuar con los estudios en relación a la aplicación de la ED en los diferentes contextos de utilización; además de los métodos y técnicas empleadas. Este artículo brinda información relevante frente a la temática de evaluación de desempeño y sus efectos en la calidad de vida laboral del trabajador, permitiendo una aproximación más detallada del tema y conociendo por cada sector las diferentes técnicas y métodos que pueden emplearse en la organización conforme sean sus objetivos; de igual manera, se invita a futuros investigadores a profundizar más, principalmente en los métodos y técnicas empleadas.

\section{Conflicto de intereses}

Los autores declaran que no existen conflictos de intereses.

\section{Referencias}

1. Cabrera I. Gestión del talento humano y el compromiso organizacional de los docentes de la maestría en Administración de la Educación en la Universidad César Vallejo, sede Huaral, 2014. Open Journal Systems. 2015;13(15):333-355. doi: 10.21503/lex.v13i15.727

2. Montoya C. Evaluación del Desempeño como Herramienta para el Análisis del Capital Humano. Visión de futuro [Internet]. 2009;1(11):1-22. Available from: https://www.redalyc.org/pdf/3579/357935472005.pdf

3. Acosta G. Gestión de Recursos Humanos en la Administración Tributaria Venezolana. Observatorio Laboral Revista Venezolana [Internet]. 2008;1(2):79-100. Available from: http://www.redalyc.org/articulo.oa?id=219016822005

4. Sánchez J, Calderón V. Diseño del proceso de evaluación del desempeño del personal y las principales tendencias que afectan su auditoría. Pensamiento y gestión. 2012;32:54-82.

5. Karkoulian S, Assaker G, Hallak R. An empirical study of 360degree feedback, organizational justice, and firm sustainability. Journal of Business Researh. 2016;69(5):1-6. doi: 10.1016/j.jbusres.2015.10.070

6. Pedraza E, Amaya G, Conde M. Desempeño laboral y estabilidad del personal administrativo contratado de la Facultad de Medicina de la Universidad de Zulia. Revista de Ciencias Sociales [Internet]. 2010;16(3):493-505. Available from:

http://www.scielo.org.ve/scielo.php?script=sci_arttext\&pi $\mathrm{d}=$ S1315-95182010000300010

7. Guelgua V, Montilla R. Importancia y características de la Gestión del Talento Humano en las medianas y grandes empresas de la ciudad de Pasto, año 2013. [Tesis de pregrado]. San Juan de Pasto, Colombia: Universidad de Nariño; 2013.

8. Gómez M. Calidad de vida laboral en empleados temporales del Valle de Aburrá-Colombia. 2010;18(24):225-236.

9. Decotiis T, Petit A. The Performance Appraisal Process: A Model and Some Testable Propositions. Academy of Management. 1978;3:635-46. doi: 10.5465/AMR.1978.4305904

10. Bontigui M. La Evaluación del Desempeño: Análisis, retos y propuestas. Una aplicación a la Comunidad Autónoma de Aragón. Revista Aragonesa de Administración Pública. 2011;(13):297-320.

11. Espinilla M, De Andrés R, Martínez F, Martínez L. A 360degree performance appraisal model dealing with heterogeneous information and dependent criteria. Information Science. 2013;222:459-471. doi: 10.1016/j.ins.2012.08.015

12. Martínez R. La evaluación del desempeño. Papeles del Psicólogo. 2010;31(1):85-96.

13. Van Dijk D, Schodl M. Performance Appraisal and Evaluation. En James D. Wright Ed. International Encyclopedia of the Social \& Behavioral Sciences, 2nd edition, Vol 17. Oxford: Elsevier; 2015. p. 716-721. doi: 10.1016/B978-0-08-097086-8.22034-5

14. Fernández M. La evaluación de desempeño, la percepción de justicia y las reacciones de los empleados. Contabilidad y Negocios [Internet]. 2009;4(8):42-48. Available from: http://revistas.pucp.edu.pe/index.php/contabilidadyNego cios/article/view/936 
15. Salgado J. Cabal Á. Evaluación del Desempeño en la Administración Pública del Principado de Asturias: Análisis de las Propiedades Psicométricas. Revista de Psicología del Trabajo y de las Organizaciones. 2011;27(2):75-91.

16. Olivera D, Cano M. La evaluación del desempeño a nivel municipal. Revista Ciencia Administrativa [Internet]. 2012;(2):117-121. Available from: https://www.uv.mx/iiesca/files/2013/04/13CA201202.p df

17. Martínez G, Guevara A. La Evaluación del Desempeño Docente. Ra Ximhai [Internet]. 2015;11(4):113-124. Available http://www.redalyc.org/pdf/461/46142596007.pdf

18. Arriche M. Evaluación de desempeño del Talento Humano de la Universidad de Fermín Toro utilizando herramientas de gestión tecnológica. Campus Virtual [Internet]. 2014;1(1):51-68. Available from: http://www.uft.edu.ve/campusvirtual/edicion1/Articulo4. pdf

19. Urdaneta O, Urdaneta M. Evaluación del desempeño y motivación del personal en los Institutos de Investigaciones de Salud. Revista de Ciencias Sociales. 2013;19(4):672-682.

20. Lasso R. Evaluación de desempeño en los colaboradores del área de producción en la empresa Industrial Colombiana de Maderas Inducolma S.A.S. [Tesis de pregrado]. Santiago de Cali, Colombia: Universidad Autónoma de Occidente; 2014.

21. Popo F. Evaluación de desempeño: Oportunidad o Amenaza. [Tesis de pregrado]. Santiago de Cali, Colombia: Universidad ICESI; 2015.

22. Valdés M, Garza R, Pérez I, Gé M, Chávez A. Una propuesta para la evaluación del desempeño de los trabajadores apoyada en el uso de técnicas cuantitativas [Internet]. Ingeniería Industrial [Internet]. 2015;36(1):48-57. Available from: http://scielo.sld.cu/pdf/rii/v36n1/rii06115.pdf

23. Islami $\mathrm{X}$, Mulolli E, Mustafa N. Using Management by Objectives as a performance appraisal tool for employee satisfaction. Future Business Journal. 2018;4(1):94-108. doi: 10.1016/j.fbj.2018.01.001

24. Selvarajan R, Cloninger P. The Importance of Accurate Performance Appraisals For Creating Ethical Organizations. The Journal of Applied Business Research. 2008;24(3):3944. doi: $10.19030 /$ jabr.v24i3.1340

25. Aguilar A, Gibert M, Oria M. El desempeño de los profesionales de Enfermería. Revista Cubana de Enfermería. 2016;32(1):87-97

26. Carlson H. A Model of Quality of Work Life as a Developmental Process. Education + Training. 1983;25(1):27-32. doi: 10.1108/eb016949

27. Bonilla CP, Franco SA, Trinidad AS. Calidad de vida laboral de conductores de pasaje foráneo. Revista Cubana de Salud y Trabajo. 2012;13(2):11-6.

28. Huerta PC, Pedraja LM, Contreras SE, y col. Calidad de vida laboral y su influencia sobre los resultados empresariales. Revista de Ciencias Sociales (Ve). 2011;17(4):658-676.

29. Zohurul I, Siengthai S. Quality of Work Life and Organizational Performance: Empirical Evidence from Dhaka Export Processing Zone. Ginebra: Conferencia Internacional Oficina de Trabajo; 2009.

30. Gabriel C, Antonieto, M, Rossi F, Bernardes A, Miguelaci T, Prado M. Utilización de indicadores de desempeño en servicio de enfermería en hospital público. Rev Latino-Am. Enfermagen. 2011;15(5):1-9.
31. Morán J. La evaluación del desempeño o de las competencias en la práctica clínica. 1ra Parte: principios y métodos, ventajas y desventajas. Educación Médica. 2016;17(4):130139. doi: 10.1016/j.edumed.2016.07.001

32. Matabajoy-Montilla JM, Matabachoy-Tulcán SM, ObandoGuerrero LM. Procesos de desarrollo del talento humano en una clínica de especialidades de Pasto-Colombia. Univ. Salud. 2018;20(1):26-36. doi: 10.22267/rus.182001.106

33. Salas R. Propuesta de estrategia para la evaluación del desempeño laboral de los médicos en Cuba. Revista Cubana de Educación Médica Superior. 2010;24(3):387-417.

34. Fernández D, Sotolongo M, Martínez C. Procedimiento para la evaluación del desempeño del personal docente en las universidades públicas angolanas. Ingeniería industrial [Internet]. 2016;37(2):178-189. Available from: http://scielo.sld.cu/scielo.php?script=sci_arttext\&pid=S18 15-59362016000200007

35. Fernández DJ, Sotolongo M, Martínez CC. La Evaluación del Desempeño por Competencias: Percepciones de Docentes y Estudiantes en la Educación Superior. Formación Universitaria [Internet]. 2016;9(5):15-24. Available from: http://www.redalyc.org/articulo.oa?id=373547712003

36. De Chaparro G, Romero L, Rincón E, Jaime H. Evaluación de desempeño docente. Cuadernos de Lingüística Hispánica. 2008;(11):167-178.

37. Fernández J, Fernández M. Elaboración de una escala de evaluación de desempeño para educadores sociales en centros de protección de menores. Intangible Capital. 2013;9(3):571-589. doi: 10.3926/ic.410

38. Piña J. La evaluación al desempeño académico. Perfiles Educativos. 2013;35(141):3-6.

39. Cárdenas M, Méndez L, González M. Evaluación del desempeño docente, estrés y burnout en profesores universitarios. Actualidades Investigativas en Educación [Internet]. 2013;14(1):1-22. Available from: https://revistas.ucr.ac.cr/index.php/aie/article/view/132 $10 / 18332$

40. Sarsosa-Prowesk K, Charria-Ortiz VH. Estrés laboral en personal asistencial de cuatro instituciones de salud nivel III de Cali, Colombia. Univ. Salud. 2018;20(1):44-52. doi: $10.22267 /$ rus.182001.108

41. Hamui A, Ortiz A, Cejudo L, Lavalle C, Vilar P. La evaluación de los docentes desde la perspectiva de los médicos residentes del Plan Único de Especializaciones Médicas. Educación Médica. 2017;18(2):89-97. doi: 10.1016/j.edumed.2016.06.010

42. Celis E, Dehesa E, Martínez A. El residente como evaluador del desempeño docente en las especialidades médicas. Educación Médica. 2017;176:1-6. doi: 10.1016/j.edumed.2017.03.021

43. Sánchez $P$, Tejada $S$. La evaluación del desempeño preprofesional: una vía para la convergencia entre dos contextos, el académico y el socioprofesional. Revista Universidad y Sociedad [Internet]. 2012;4(1):1-7. Available from: https://rus.ucf.edu.cu/index.php/rus/article/view/339/8 03

44. Sapién A, Gutiérrez M, Piñón L, Araiza P. Evaluación del desempeño por el método de 360 grados y por factores de evaluación en una empresa mexicana. Publicaciones. 2016;46:13-29.

45. Dizaji M, Mazdeh M, Makui A. Performance evaluation and ranking of direct sales stores using BSC approach and fuzzy multiple attribute decision-making methods. Decision 
Science Letters. 2018;7(2):197-210. doi: 10.5267/j.dsl.2017.5.003

46. Manjarrés A, Castell R, Luna C. Modelo de Evaluación del Desempeño Basado en Competencias. Ingeniare. 2013;8(15):11-29.

47. Alves Corrêa D, Cirera Oswaldo Y, Carlos Giuliani A. Vida con calidad y calidad de vida en el trabajo. Invenio [Internet]. 2013;16(30):145-163. Disponible en: http://www.redalyc.org/articulo.oa?id=87726343010

48. López J, Barahona N, Estrada, M, y col. Evaluación de competencias del profesional de enfermería que labora en hospitales públicos y privados. Revista Enfermería Actual en Costa Rica. 2014;27:1-15.

49. Alarcón J. Mejora del Sistema de Evaluación del Desempeño Laboral en la Rama Ejecutiva del Poder Público en Colombia. [Tesis de maestría]. Santiago de Compostela, España: Universidad de Santiago de Compostela; 2015.

50. Vázquez E. La evaluación del desempeño en las grandes empresas Españolas. Universia Business Review [Internet]. 2007; (15): 42-53. Available from: https://ubr.universia.net/article/viewFile/609/735

51. Oktay C, Senol Y, Rinnert S, Cete Y. Utility of 360-degree assessment of residents in a Turkish academic emergency medicine residency program. Turkish Journal of Emergency Medicine. 2017;17(1):12-15. doi: 10.1016/j.tjem.2016.09.007

52. Elizalde L, Reyes R. Elementos clave para la evaluación del desempeño de los docentes. Revista Electrónica de Investigación Educativa [Internet]. 2008;10(Esp):1-13. Available from: https://redie.uabc.mx/redie/article/view/198/1272

53. Castillo L. Consideraciones para la evaluación del desempeño del personal del sector público de salud chileno. Revista Gestión de las Personas y Tecnología [Internet].
2017;10(28):5-19. Available from: http://www.revistas.usach.cl/ojs/index.php/revistagpt/ar ticle/viewFile/2851/2589

54. Bitran M, Mena B, Riquelme A, Padilla O, Sánchez I, Moreno R. Desarrollo y validación de un instrumento en español para evaluar el desempeño de docentes clínicos a través de las percepciones de sus estudiantes. Revista Médica de Chile. 2010;138(6):685-693.

55. Rueda M. La evaluación del desempeño docente en la Universidad. Revista Electrónica de Investigación Educativa [Internet]. 2008; 10(Esp):1-15. Available from: http://www.redalyc.org/pdf/155/15511127002.pdf

56. Rowland C. Organizational justice and performance: is appraisal fair? EuroMed Journal of Busines. 2012;7(3):280293. doi: $10.1108 / 14502191211265334$

57. Doanis L. Performance Appraisal System: It's Implication To Employee Performance. International Journal of Economics and Management Science [Internet]. 2012;2(3):55-62. Available from: https://pdfs.semanticscholar.org/c716/802af3a90064ca0 dc196578574e07b5e79df.pdf

58. Sikes D, Jestes E, LeClair C, Yates R. Using a $360^{\circ}$ Feedback Evaluation to Enhance Interprofessional Collaboration. Nurse Leader. 2015;13(4):64-69. doi: 10.1016/j.mnl.2014.10.002

59. Del Toro C, Guzmán M, Pérez R. La evaluación del desempeño, los procesos y la organización. Ingeniería Industrial [Internet]. 2016;37(2):164-177. Available from: http://scielo.sld.cu/pdf/rii/v37n2/rii06216.pdf

60. Mihalcea A, Mihalcea D. Management Skills Assessment Usin $360^{\circ}$ Feedback - Msf 360. Procedia-Socialand Behavioral Science. 2015;187(13):318-323. doi: 10.1016/j.sbspro.2015.03.059 